\title{
Fabrication of a wafer-scale uniform array of single-crystal organic nanowire complementary inverters by nanotransfer printing
}

\author{
Kyung Sun Park, Jangmi Baek, Yong-Eun Koo Lee and Myung Mo Sung*
}

\begin{abstract}
We report the fabrication and electrical characterization of a wafer-scale array of organic complementary inverters using single-crystal 6,13-bis(triisopropylsilylethynyl) pentacene (TIPS-PEN) and fullerene $\left(C_{60}\right)$ nanowires as $p$ - and $n$-channels, respectively. Two arrays of single-crystal organic nanowires were generated consecutively on desired locations of a common substrate with a desired mutual alignment by a direct printing method (liquid-bridge-mediated nanotransfer molding). Another direct printing of silver micron scale structures, as source and drain electrodes, on the substrate with the two printed nanowire arrays produced an array of complementary inverters with a bottom gate, top contact configuration. Field-effect mobilities of single-crystal TIPS-PEN and $\mathrm{C}_{60}$ nanowire field-effect transistors (FETs) in the arrays were uniform with $1.01 \pm 0.14$ and $0.10 \pm 0.01 \mathrm{~cm}^{2} V^{-1} \mathrm{~s}^{-1}$, respectively. A wafer-scale array of complementary inverters produced all by the direct printing method showed good performance with an average gain of 25 and with low variations among the inverters.
\end{abstract}

Keywords: Single-crystal organic nanowires; Direct printing; Field-effect transistors; Complementary inverters

\section{Background}

Organic field-effect transistors (OFETs) are widely studied as a basic device component for a variety of applications such as displays, memory devices and sensors [1-4]. OFETs fabricated from organic single-crystals, in particular, are of great interest as they have shown the best OFET performance with the highest charge carrier mobility due to the perfect order of molecules and the absence of grain boundaries within the crystal structures [5-8]. Highperformance $p$ - and $n$-channel OFETs may allow for fabrication of high-performance organic complementary integrated circuits for logic operation such as a complementary inverter [9-11]. It should be noted that constructing a complementary inverter is technically complex because both $p$ - and $n$-channel transistors are required to be patterned onto a common substrate in a desired alignment to each other. Especially, single-crystal organic complementary circuits are difficult to fabricate as it involves manipulation of organic crystals which are typically nanosized, fragile and brittle [12,13]. In order to avoid such difficulties of manual positioning of each crystal, several

* Correspondence: smm@hanyang.ac.kr

Department of Chemistry, Hanyang University, Seoul 133-791, Korea approaches have been employed to prepare the inverters. In one approach, inverters were fabricated on a single piece of ambipolar crystal, and $p$ - and $n$-channel FETs were realized by using both low and high work function metals as electrodes [14-16]. In another approach, organic $p$ - and $n$-type single-crystals were separately grown and randomly casted on defined areas of a common substrate [17]. In the other approach, $p$ - and $n$-type crystals were simultaneously synthesized and positioned on a common substrate in a desired arrangement using organic crystallization methods, e.g. droplet-pinned crystallization (DPC), electrohydrodynamic printing and liquid-bridgemediated nanotransfer molding (LB-nTM) $[9,18,19]$.

Herein we adopted the LB-nTM approach for fabricating arrays of single-crystal OFETs and complementary inverters by employing 6,13-bis(triisopropylsilylethynyl) pentacene (TIPS-PEN) and fullerene $\left(\mathrm{C}_{60}\right)$ as $p$-type and $n$-type materials, respectively. The organic semiconductor wires with various widths were prepared by using molds patterned with different sized channels, and characterized for their crystallinities. Single-crystal structures were observed only for the organic semiconductors produced in the nanochannels of the width around $100 \mathrm{~nm}$ 
or less, and the height of about $150 \mathrm{~nm}$. A p-type fieldeffect mobility of $1.01 \pm 0.14 \mathrm{~cm}^{2} \mathrm{~V}^{-1} \mathrm{~s}^{-1}$ and an $n$-type field-effect mobility of $0.10 \pm 0.01 \mathrm{~cm}^{2} \mathrm{~V}^{-1} \mathrm{~s}^{-1}$ were obtained for arrays of OFETS based on single-crystal TIPS-PEN and $C_{60}$ nanowires, respectively. By electrically combining the $p$-type TIPS-PEN and $n$-type $\mathrm{C}_{60}$ device arrays, we demonstrate the operation of an array of organic single-crystal complementary inverters with a high and uniform gain of $\sim 25$.

\section{Methods}

\subsection{Materials}

The TIPS-PEN ink solution was prepared by dissolving TIPS-PEN (2 wt\%, $\geq 99 \%$, Sigma Aldrich Inc.) in 1,2,3,4tetrahydronaphthalene (Tetralin, $\geq 97 \%$, Sigma Aldrich Inc.) solvent. The $\mathrm{C}_{60}$ ink solution was prepared by dissolving $\mathrm{C}_{60}$ powder (1 wt\%, 99.5\%, Sigma Aldrich Inc.) in 1,3,5-trichlorobenzene (TCB, 99\%, Sigma Aldrich Inc.). The Ag nanoparticle ink (DGP 40LT-15C) was purchased from Advanced Nano Products. The Ag ink contains silver nanoparticles (20 wt\%, particle diameter $40-50 \mathrm{~nm}$ ) dispersed in methanol solvent. Polyurethane acrylate (PUA) (MINS-ERM, Minuta Tech.) was used to prepare the UVcurable hard molds for producing organic semiconductor wires. Polydimethylsiloxane (PDMS) (Sylgard 184, Dow Corning) was used to prepare thermal-curable molds for producing silver microelectrodes.

\subsection{Preparation of substrates}

As a typical transferring substrate, the $\mathrm{Si}$ substrates, cut from $n$-type (100) wafers with resistivity in the range of 1-5 $\Omega \cdot \mathrm{cm}$, were used. The Si substrates were initially treated by the chemical cleaning process, proposed by Ishizaka and Shiraki [20], which involves degreasing, $\mathrm{HNO}_{3}$ boiling, $\mathrm{NH}_{4} \mathrm{OH}$ boiling (alkali treatment), $\mathrm{HCl}$ boiling (acid treatment), rinsing in deionized water, and blow-drying with nitrogen. A thin oxide layer was grown by placing the $\mathrm{Si}$ substrate in a piranha solution (4:1 mixture of $\mathrm{H}_{2} \mathrm{SO}_{4}: \mathrm{H}_{2} \mathrm{O}_{2}$ ) for 10-15 min. The substrate was rinsed several times with deionized water (resistivity $=18$ $\mathrm{M} \Omega \cdot \mathrm{cm}$ ), then dried with a stream of nitrogen. For the electrical measurements, a heavily doped $p$-type $\mathrm{Si}$ (0.001-0.003 $\Omega \mathrm{cm}^{-1}$, Hissan Inc.) with a $200 \mathrm{~nm}$ thick, thermally grown $\mathrm{SiO}_{2}$ layer was used as a thick oxide substrate.

\subsection{Growth of single-crystal organic nanowires}

Arrays of organic semiconductors were made on an oxidized Si substrate using the LB-nTM method with PUA molds [21]. The PUA molds were fabricated by casting PUA on the masters that are silicon wafers with desired line patterns made by e-beam/photo lithography. In this study four different line patterns were used for the masters - the width of the parallel lines and the spaces between the lines were $50 \mathrm{~nm} / 150 \mathrm{~nm}, 100 \mathrm{~nm} / 100 \mathrm{~nm}$, $500 \mathrm{~nm} / 200 \mathrm{~nm}$ and $10 \mu \mathrm{m} / 10 \mu \mathrm{m}$, respectively. After UV curing ( $10 \mathrm{~min})$, the PUA molds were peeled away from the masters.

In order to generate arrays of organic semiconductors, only recessed channels of the patterned PUA mold were filled with a TIPS-PEN or $\mathrm{C}_{60}$ ink solution using discontinuous dewetting [22]. By dragging a dropped ink solution over the patterned mold with a glass stick or a needle, the meniscus of the ink solution moved over the surface of the mold to fill the channels without leaving any residue on the raised surface. The ink in the channels was next solidified by drying at mild temperatures below $100^{\circ} \mathrm{C}$ for $30-60 \mathrm{~min}$. The mold containing the dried organic semiconductors was then brought into contact with a substrate surface covered by a thin ethanol layer. The ethanol layer on the substrate formed a liquid bridge (a capillary bridge) between the substrate and the mold. The liquid bridge allowed good conformal contact between the semiconductors contained in the mold and the substrate. As the ethanol evaporated, the attractive capillary force gradually increased, pulling the two surfaces into contact, and providing good conformal contact between them. After drying, separation of the mold from the substrate yielded an organic semiconductor array on the substrate.

\subsection{Fabrication of a wafer-scale array of complementary inverters}

The fabrication of an array of single-crystal organic nanowire inverters was initiated by generation of a singlecrystal TIPS-PEN nanowire array, as active $p$-channels, on $200 \mathrm{~nm}$-thick $\mathrm{SiO}_{2} / \mathrm{p}^{+}$-Si substrates using LB-nTM. Subsequently, an array of single-crystal $\mathrm{C}_{60}$ nanowires as active $n$-channels was placed alongside the TIPS-PEN nanowire array using LB-nTM. Next, on this substrate with the two printed nanowire arrays, source and drain electrodes of $1.5 \mu \mathrm{m}$-thick Ag were prepared and placed by LB-nTM so as to electrically connect the arrays of $p$-type and $n$-type FETs, producing an array of complementary inverters.

Here, we used molds of different materials and specially designed patterns for printing organic semiconductor nanowires and Ag microelectrodes. The two semiconductor arrays were prepared using a wafer-scale PUA mold that contains 180 sections of a line pattern-each section contains 50 nanochannels of $100 \mathrm{~nm}$ width, $10 \mu \mathrm{m}$ length and $200 \mathrm{~nm}$ depth-with the space between the sections being $2 \mathrm{~mm}$. For the Ag electrodes, a wafer-scale PDMS mold was fabricated by casting PDMS on the master which is a silicon wafer with an embossed pattern-composed of 180 basic units-made by photolithography. After curing at $70^{\circ} \mathrm{C}$ for $50 \mathrm{~min}$, the PDMS mold was peeled away from the master so as to be used for printing Ag electrodes. 


\subsection{Characterization}

The samples were characterized using a scanning electron microscope (SEM, Hitachi S4800) operated at $15 \mathrm{kV}$. The crystallinity of the organic semiconductors was examined by selective-area electron diffraction (EM 912 Omega) with a transmission electron microscope run at $120 \mathrm{kV}$. All current-voltage $(I-V)$ properties of the FETs and inverters were measured with a semiconductor parameter analyzer (HP 4155C, Agilent Technologies) in the dark and in ambient air (relative humidity $45 \%$ ) at $20^{\circ} \mathrm{C}$. The field-effect mobility $(\mu)$ and threshold voltage $\left(V_{\text {th }}\right)$ were calculated in the saturation regime $\left(\mathrm{V}_{\mathrm{DS}}=-50\right.$ or $\left.50 \mathrm{~V}\right)$ by plotting the square root of the drain current $\left(\mathrm{I}_{\mathrm{DS}}\right)$ versus the gate voltage $\left(\mathrm{V}_{\mathrm{GS}}\right)$ using $\mathrm{I}_{\mathrm{DS}}=\left(\mathrm{WC}_{i} / 2 \mathrm{~L}\right) \mu\left(\mathrm{V}_{\mathrm{GS}}-\mathrm{V}_{\mathrm{th}}\right)^{2}$, where $C_{i}$ is the capacitance per unit area of the gate dielectric layer, and $\mathrm{W}$ and $\mathrm{L}$ are the channel width and length, respectively.

\section{Results and discussion}

Single-crystal organic semiconductor nanowires were fabricated in arrays on Si substrates by LB-nTM using 6,13-bis(triisopropylsilylethynyl) pentacene (TIPS-PEN) and fullerene $\left(\mathrm{C}_{60}\right)$ as $p$-type and $n$-type materials, respectively. Figure 1(a) illustrates the procedure used to prepare an organic semiconductor array by LB-nTM. Organic molecular ink solutions fill only the channels of the PUA mold due to selective inking based on discontinuous dewetting [22]. The inks inside the channels are then dried into solid forms at mild temperature $\left(<100^{\circ} \mathrm{C}\right)$. The mold with solidified organic semiconductors is brought into contact with a substrate whose surface is covered by a thin polar liquid layer. After drying, separation of the mold from the substrate yields an organic semiconductor array on the substrate. In the LB-nTM approach, crystallinity of the materials formed inside the channels is believed to depend on the dimension of the channels [21]. According to previous LB-nTM studies, nanowires having a width of $90-110 \mathrm{~nm}$ and a height of about $130 \mathrm{~nm}$ were single crystals while microribbons having a width of $2 \mu \mathrm{m}$ were of semi-crystalline nature [21]. Here, we prepared TIPS-PEN wires by employing line patterns of four different dimensions-each having the channels with a width of $50 \mathrm{~nm}, 100 \mathrm{~nm}$, $500 \mathrm{~nm}$ and $10 \mu \mathrm{m}$, respectively and a height of about $150 \mathrm{~nm}$ for all cases-so as to study in details the relationship between the channel sizes and the crystallinity of the produced organic semiconductors. Figures 1(b)-(e) show the scanning electron microscope (SEM) images and their corresponding selective-area electron diffraction (SAED) patterns of four different TIPS-PEN arrays, each consisting of $50 \mathrm{~nm}, 100 \mathrm{~nm}, 500 \mathrm{~nm}$ and $10 \mu \mathrm{m}$ wide TIPS-PEN wires, respectively.

In the SAED pattern for the semiconductors prepared in $10 \mu \mathrm{m}$ wide channels, several diffraction spots appear
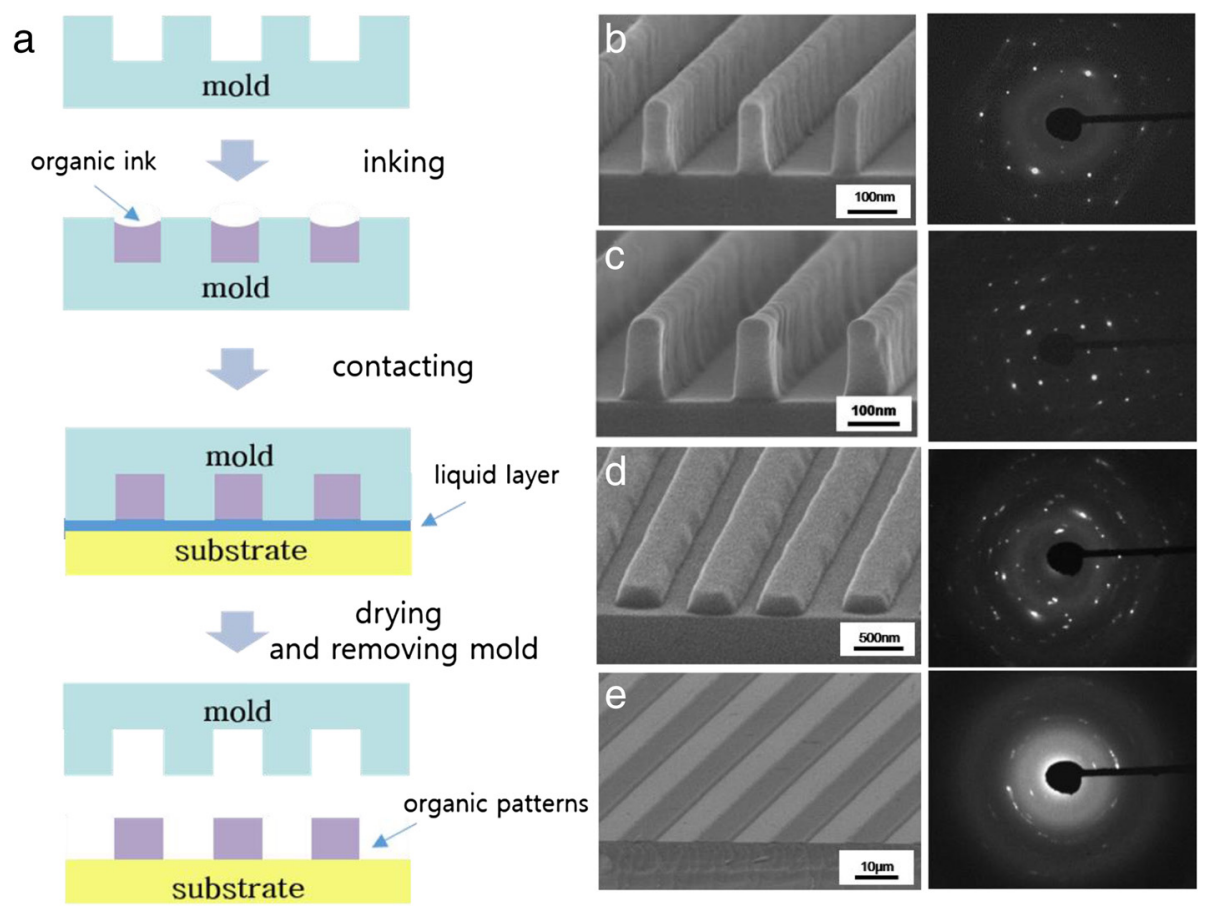

Figure 1 Fabrication of organic wire array using LB-nTM. (a) Schematic illustration of the procedure used to fabricate an organic semiconductor array on a substrate using LB-nTM. (b)-(e) SEM images and corresponding SAED patterns of $50 \mathrm{~nm}, 100 \mathrm{~nm}, 500 \mathrm{~nm}$, and $10 \mu \mathrm{m}$ wide TIPS-PEN wires on the Si substrate, respectively. 
in a couple of Debye rings, which indicates the polycrystalline nature. For the semiconductors prepared in $500 \mathrm{~nm}$ wide channels, many diffraction spots are observed and they form a grid-like pattern, which indicates the semi-crystalline nature. For the TIPS-PEN semiconductors prepared in $100 \mathrm{~nm}$ or $50 \mathrm{~nm}$ width, the SAED patterns clearly present very well-ordered diffraction spots, indicating that they are of single-crystalline nature. These results confirm that the nano confinement effect provided by the nanochannels in the molds is crucial for the formation of the single-crystal TIPS-PEN nanowires in the LB-nTM method. More specifically, these results suggest that both the width and the height of the cross sections of the channels should be around $100 \mathrm{~nm}$ or less in order to have single-crystal structures.

A large-scale array of complementary inverters made of single-crystal TIPS-PEN and $\mathrm{C}_{60}$ organic nanowires was prepared on a 2-inch Si wafer as shown in Figure 2(a). The inverter array was fabricated by direct printing through LB-nTM as demonstrated in a schematic illustration that focuses on a single complementary inverter (Figure 2(b)). First, a single-crystal TIPS-PEN nanowire array was generated on a Si substrate as active $p$-channels using LB-nTM. Subsequently, an array of single-crystal $\mathrm{C}_{60}$ nanowires as active $n$-channels was placed alongside the TIPS-PEN nanowire using LB-nTM. Next, a pair of $1.5 \mu \mathrm{m}$-thick Ag structures with $3 \mu \mathrm{m}$ gap between them were defined as source and drain electrodes to contact the TIPS-PEN nanowires and the $\mathrm{C}_{60}$ nanowires on the substrate by LB-nTM so as to prepare the arrays of $p$-type and $n$-type OFETs as well as the complementary inverters.

The structure of each complementary inverter in the array is schematically illustrated along with SEM images for two OFETs that comprise the inverter (Figure 2(c)). Each OFET contains 50 nanowires and a pair of silver electrodes that are separated by a distance of $3 \mu \mathrm{m}$, and therefore has an active channel of micro-scale dimension, $5 \mu \mathrm{m}$ (100 $\mathrm{nm}$ wide nanowire $\times 50$ ea) width and $3 \mu \mathrm{m}$ length. The array contains a total of 180 complementary inverters. Notably, fabrication of such large-scale array was accomplished by simply direct printing of different materials three times using molds of specially designed patterns.

Representative transfer and output curves for the two single-crystal organic nanowire FETs show that these OFETs have well-defined transistor characteristics with saturation effects (Figure 3(a), (b)). We can calculate the
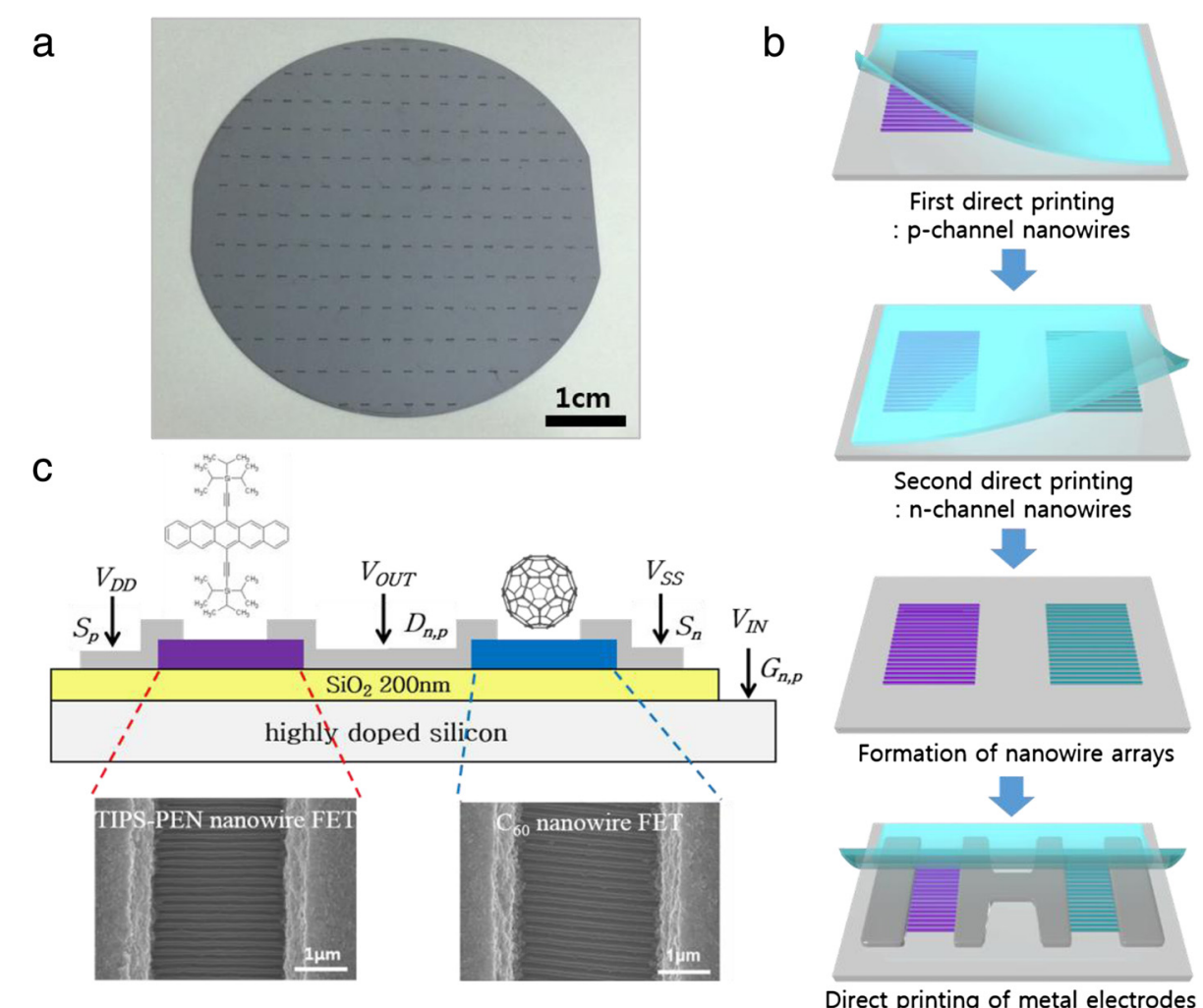

Figure 2 Fabrication of organic complementary inverters using LB-nTM. (a) A photography of a large-scale array of complementary inverters made of single-crystal TIPS-PEN (p-type) and C60 (ntype) organic nanowires on a 2-inch Si wafer. (b) Schematic illustration of the procedure used to fabricate a complementary inverter by LB-nTM. (c) Schematic representations and SEM images of positioning p-type and n-type single-crystal nanowires with Ag electrodes. 
electrical parameters of each OFET from these curves. The $p$-type transistor with fifty TIPS-PEN nanowires was found to have a field-effect mobility of $1.1 \mathrm{~cm}^{2} \mathrm{~V}^{-1} \mathrm{~s}^{-1}$ in the saturation region at $\mathrm{V}_{\mathrm{DS}}=-50 \mathrm{~V}$, an on/off current ratio of $\sim 10^{5}$, and a threshold voltage of $-9.3 \mathrm{~V}$. For comparison, OFET with one $10 \mu \mathrm{m}$ wide TIPS-PEN microribbons was also characterized to show a field-effect mobility of $0.01 \mathrm{~cm}^{2} \mathrm{~V}^{-1} \mathrm{~s}^{-1}$ at $\mathrm{V}_{\mathrm{DS}}=-50 \mathrm{~V}$, an on/off current ratio of $\sim 10^{4}$, and a threshold voltage of $-3.8 \mathrm{~V}$ (Additional file 1: Figure S1). The field-effect mobility from singlecrystal organic nanowire FETs was enhanced compared to that from polycrystalline microribbon FETs, which is probably due to highly ordered molecules within their crystals as we demonstrated the dependence of crystallinity according to their structure dimension in Figure 1. For the $n$-type transistor with fifty $C_{60}$ nanowires, we also extracted a field-effect mobility of $0.12 \mathrm{~cm}^{2} \mathrm{~V}^{-1} \mathrm{~s}^{-1}$ in the saturation region at $\mathrm{V}_{\mathrm{DS}}=50 \mathrm{~V}$, an on/off current ratio of $\sim 10^{3}$, and a threshold voltage of $8.2 \mathrm{~V}$ from Figure 3a. We extended our investigation to the arrays of 180 OFETs with TIPS-PEN and $\mathrm{C}_{60}$ nanowires, respectively, which shows remarkably uniform distributions of mobilities, as shown in Figure 3(c), (d). The average values and standard deviations of hole mobilities for the TIPS-PEN OFETs in the array are $1.01 \pm 0.14 \mathrm{~cm}^{2} \mathrm{~V}^{-1} \mathrm{~s}^{-1}$ and those of electron mobilities for the $C_{60}$ OFETs in the array are $0.10 \pm$ $0.01 \mathrm{~cm}^{2} \mathrm{~V}^{-1} \mathrm{~s}^{-1}$, respectively. We note that the variation in mobility values is commonly seen in a device array, which may be attributed to variable morphology and crystallinity of active channels [23,24]. The observed high uniformity in mobilities of the two OFETs in the arrays indicates that the crystalline nanowires produced in an array by LB-nTM are of almost identical quality.

As a consequence of the two arrays of $p$-type and $n$-type OFETs having uniform mobility values, fabrication of an array of complementary inverters-with uniformity in both their size/structure as well as their performance-was realized. Figure 4(a) demonstrates a typical voltage-transfer curve of the complementary inverters prepared from single-crystal $p$-type TIPS-PEN and $n$-type $\mathrm{C}_{60}$ nanowires. Here, a common gate functioned as the input node $\left(\mathrm{V}_{\mathrm{IN}}=0-60 \mathrm{~V}\right)$ while the source of the driver $\left(n\right.$-channel) was grounded $\left(V_{S S}=0\right)$ and the source of the load ( $p$-channel) provided the supply voltage, $V_{D D}=60 \mathrm{~V}$ (see Figure $4 \mathrm{a}$, inset for the circuit diagram). Notably the inverter exhibits a well-
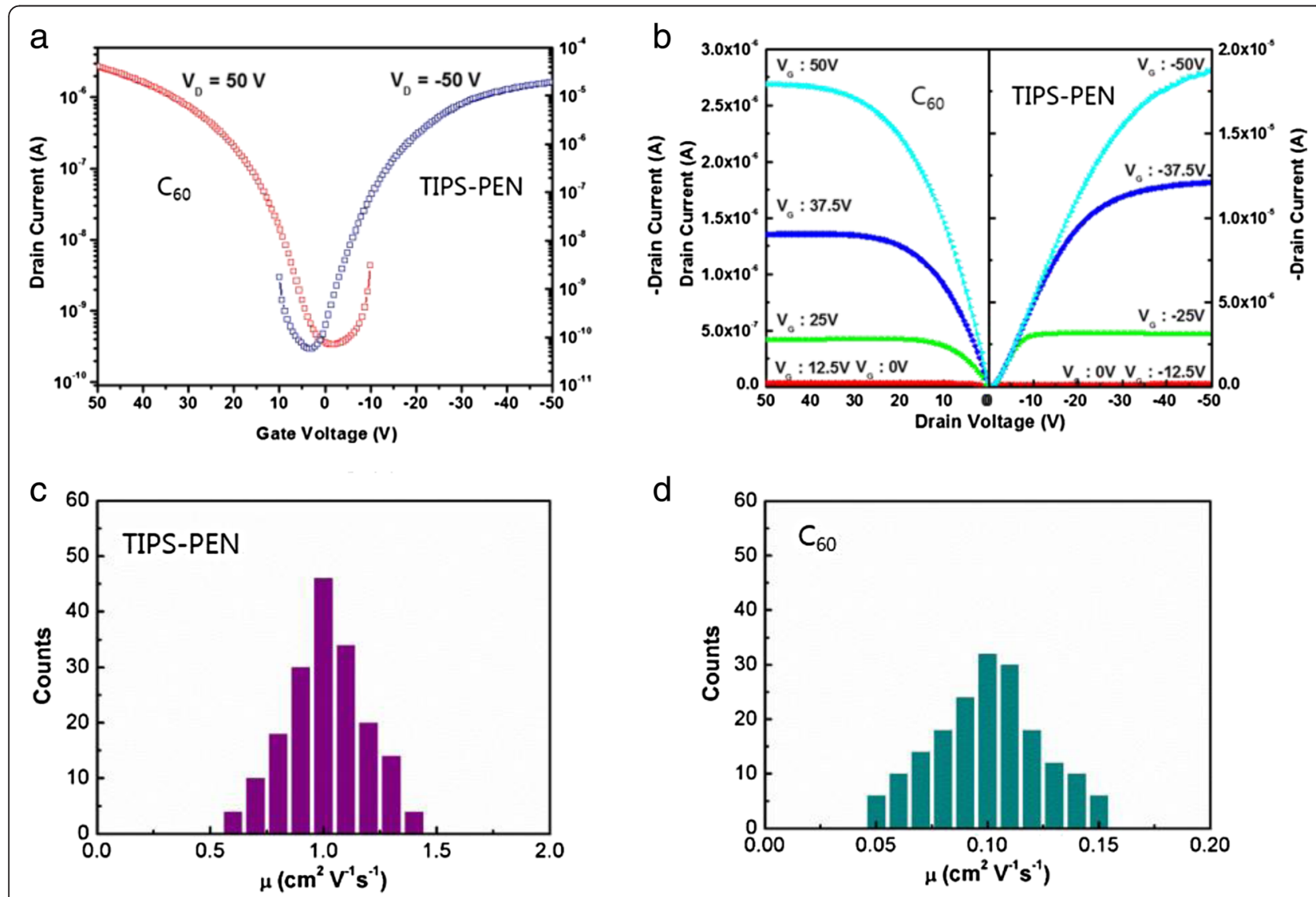

Figure $\mathbf{3}$ OFET characteristics. (a) Typical transfer and (b) output characteristics of the OFETs based on $p$-channel TIPS-PEN and $n$-channel $C_{60}$ single-crystal nanowires, respectively. (c), (d) Field effect mobility distributions for the single-crystal TIPS-PEN and $\mathrm{C}_{60}$ nanowire OFETs, respectively. 


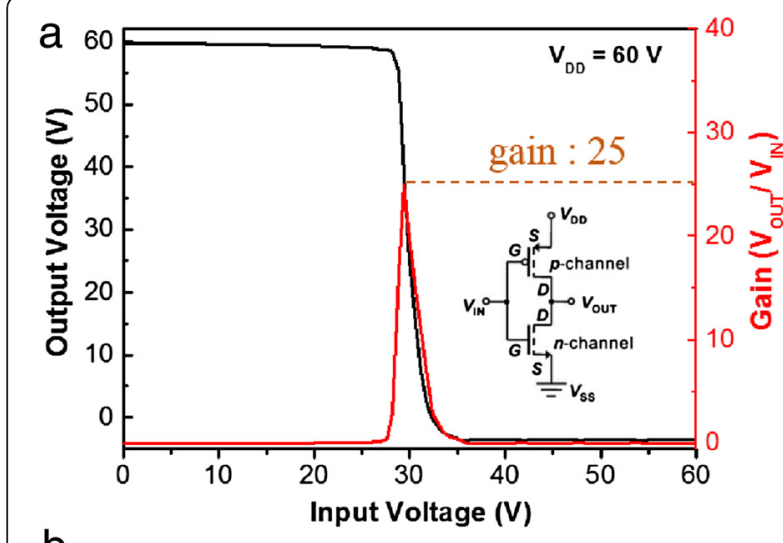

$\mathrm{b}$

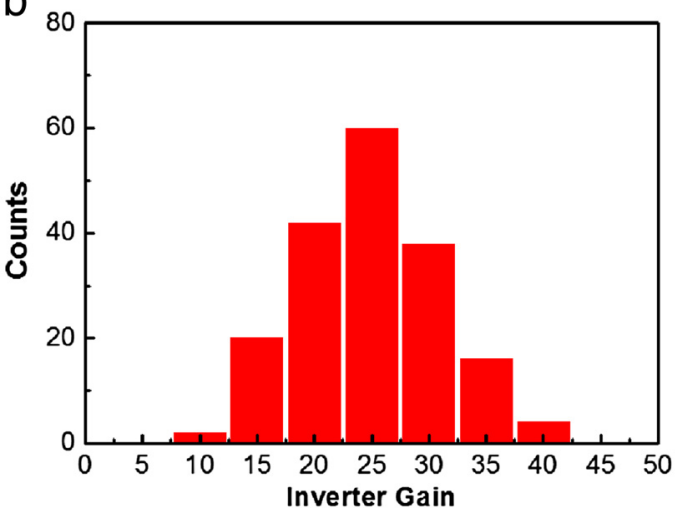

Figure 4 Inverter characteristics. (a) Transfer curve and the corresponding gain of the complementary inverter comprised of single-crystal $p$-channel TIPS-PEN and $n$-channel $C_{60}$ nanowires. (b) Voltage gain distributions of the 180 inverters produced from the single-crystal TIPS-PEN and $\mathrm{C}_{60}$ nanowire OFETs.

defined transfer curve which clearly displays the inverter's sharp switching operation, despite the large mismatch in charge carrier mobilities between the two semiconducting nanowires. The inverter threshold voltage (at $\mathrm{V}_{\text {IN }}=\mathrm{V}_{\text {OUT }}$ ) is measured to be $30 \mathrm{~V}$-which is the half of the $\mathrm{V}_{\mathrm{DD}}$-and a high gain $\left(-\mathrm{d} V_{\mathrm{OUT}} / \mathrm{d} V_{\mathrm{IN}}\right)$ of 25 is obtained as shown in Figure 4a.

Importantly, the 180 complementary inverters, each made of single-crystal TIPS-PEN and $\mathrm{C}_{60}$ nanowires, fabricated in an array form on a Si substrate, show the uniformity in their performance with a mean gain value of 24.7 and a standard deviation of 5.05 as demonstrated in Figure 4(b). The large and uniform gain of the devices suggests high promise for printed arrays of logical circuits by LB-nTM in transparent, flexible, inexpensive, and large-area electronic applications.

\section{Conclusions}

In summary, we used LB-nTM, a direct printing method, to fabricate an array of organic complementary inverters using single-crystal TIPS-PEN and $\mathrm{C}_{60}$ nanowires as $p$ - and $n$-channels, respectively, and Ag micron-sized structures as electrodes. It was found that both the width and the height of the cross sections of the channels should be around $100 \mathrm{~nm}$ or less in order to produce single-crystal structures. The OFET arrays based on the single-crystal nanowires show highly uniform fieldeffect mobilities of $1.01 \pm 0.14$ and $0.10 \pm 0.01 \mathrm{~cm}^{2} \mathrm{~V}^{-1} \mathrm{~s}^{-1}$ for TIPS-PEN and $C_{60}$, respectively. The complementary inverter array with TIPS-PEN nanowires as $p$-channels and $\mathrm{C}_{60}$ nanowires as $n$-channels showed good performance with a gain of $\sim 25$. Printed single-crystal organic nanowire arrays could potentially be used in organic nanowire integrated electronics for transparent, flexible, inexpensive, and large-area applications.

\section{Additional file}

Additional file 1: Figure S1. (a) Typical transfer and (b) output characteristics of the OFETs based on $10 \mu \mathrm{m}$ wide TIPS-PEN microribbons, respectively.

\section{Competing interests}

The authors declare no competing financial interest.

\section{Authors' contributions}

MMS conceived and designed the experiment. KSP and JB performed the experiment and analyzed the data. KSP, Y-EKL and MMS Co-wrote the paper. All authors read and approved the final manuscript.

\section{Acknowledgements}

This work was supported by the Global Frontier R\&D Program on the Center for Multiscale Energy System (no. 2011-0031562) and Nano - Material Technology Development Program (2012M3A7B4034985) through the National Research Foundation of Korea (NRF) funded by the Ministry of Science, ICT \& Future Planning, and by Samsung Research Funding Center of Samsung Electronics under Project Number SRFC-MA1401-05.

Received: 11 September 2014 Accepted: 6 October 2014 Published online: 10 February 2015

\section{References}

1. H Sirringhaus, Materials and applications for solution-processed organic field-effect transistors. Proc. IEEE 97, 1570 (2009)

2. $\mathrm{P}$ Lin, $\mathrm{F}$ Yan, Organic thin-film transistors for chemical and biological sensing. Adv. Mater. 24, 34 (2012)

3. L Zhang, D C-a, G Yu, Y Liu, Solution processed organic field-effect transistors and their application in printed logic circuits. J. Mater. Chem. 20, 7059 (2010)

4. T Someya, A Dodabalapur, J Huang, KC See, HE Katz, Chemical and physical sensing by organic field-effect transistors and related devices. Adv. Mater. 22, 3799 (2010)

5. HY Li, BCK Tee, JJ Cha, Y Cui, JW Chung, SY Lee, ZN Bao, High-mobility field-effect transistors from large-area solution-grown aligned $C_{60}$ single crystals. J. Am. Chem. Soc. 134, 2760 (2012)

6. H Minemawari, T Yamada, H Matsui, J Tsutsumi, S Haas, R Chiba, R Kumai, T Hasegawa, Inkjet printing of single-crystal films. Nature 475, 364 (2011)

7. YB Yuan, G Giri, AL Ayzner, AP Zoombelt, SCB Mannsfeld, JH Chen, D Nordlund, MF Toney, JS Huang, ZN Bao, Ultra-high mobility transparent organic thin film transistors grown by an off-centre spin-coating method. Nat. Commun. 5, 3005 (2014)

8. YH Kim, B Yoo, JE Anthony, SK Park, Controlled deposition of a highperformance small-molecule organic single-crystal transistor array by direct ink-jet printing. Adv. Mater. 24, 497 (2012)

9. HY Li, BCK Tee, G Giri, JW Chung, SY Lee, ZN Bao, High-performance transistors and complementary inverters based on solution-grown aligned organic single-crystals. Adv. Mater. 24, 2588 (2012) 
10. AL Briseno, RJ Tseng, SH Li, CW Chu, Y Yang, EHL Falcao, F Wudl, MM Ling, HZ Chen, ZN Bao, H Meng, C Kloc, Organic single-crystal complementary inverter. Appl. Phys. Lett. 89, 222111 (2006)

11. MM Ling, ZN Bao, P Erk, M Koenemann, M Gomez, Complementary inverter using high mobility air-stable perylene di-imide derivatives. Appl. Phys. Lett. 90, 093508 (2007)

12. AL Briseno, SCB Mannsfeld, SA Jenekhe, Z Bao, YN Xia, Introducing organic nanowire transistors. Mater. Today 11, 38 (2008)

13. QX Tang, YH Tong, WP Hu, Q Wan, T Bjornholm, Assembly of nanoscale organic single-crystal cross-wire circuits. Adv. Mater. 21, 4234 (2009)

14. TD Anthopoulos, DM de Leeuw, E Cantatore, S Setayesh, EJ Meijer, C Tanase, JC Hummelen, PWM Blom, Organic complementary-like inverters employing methanofullerene-based ambipolar field-effect transistors. Appl. Phys. Lett. 85, 4205 (2004)

15. T Uemura, M Yamagishi, Y Okada, K Nakayama, M Yoshizumi, M Uno, J Takeya, Monolithic complementary inverters based on organic single crystals. Adv. Mater. 22, 3938 (2010)

16. M Ahles, R Schmechel, $\mathrm{H}$ von Seggern, Complementary inverter based on interface doped pentacene. Appl. Phys. Lett. 87, 113505 (2005)

17. AL Briseno, SCB Mannsfeld, C Reese, JM Hancock, Y Xiong, SA Jenekhe, Z Bao, YN Xia, Perylenediimide nanowires and their use in fabricating fieldeffect transistors and complementary inverters. Nano Lett. 7, 2847 (2007)

18. JK Hwang, S Cho, JM Dang, EB Kwak, K Song, J Moon, MM Sung, Direct nanoprinting by liquid-bridge-mediated nanotransfer moulding. Nat. Nanotechnol. 5, 742 (2010)

19. SY Min, TS Kim, BJ Kim, H Cho, YY Noh, H Yang, JH Cho, TW Lee, Large-scale organic nanowire lithography and electronics. Nat. Commun. 4, 1773 (2013)

20. A Ishizaka, Y Shiraki, Low-temperature surface cleaning of silicon and its application to silicon MBE. J. Electrochem. Soc. 133, 666 (1986)

21. KS Park, B Cho, J Baek, JK Hwang, H Lee, MM Sung, Single-crystal organic nanowire electronics by direct printing from molecular solutions. Adv. Funct. Mater. 23, 4776 (2013)

22. RJ Jackman, DC Duffy, E Ostuni, ND Willmore, GM Whitesides, Fabricating large arrays of microwells with arbitrary dimensions and filling them using discontinuous dewetting. Anal. Chem. 70, 2280 (1998)

23. M Wang, J Li, GY Zhao, QH Wu, YG Huang, WP Hu, XK Gao, HX Li, DB Zhu, High-performance organic field-effect transistors based on single and largearea aligned crystalline microribbons of 6,13-dichloropentacene. Adv. Mater. 25, 2229 (2013)

24. V Podzorov, E Menard, A Borissov, V Kiryukhin, JA Rogers, ME Gershenson, Intrinsic charge transport on the surface of organic semiconductors. Phys. Rev. Lett. 93, (2004)

\section{Submit your manuscript to a SpringerOpen ${ }^{\circ}$ journal and benefit from:}

- Convenient online submission

- Rigorous peer review

- Immediate publication on acceptance

- Open access: articles freely available online

- High visibility within the field

- Retaining the copyright to your article

Submit your next manuscript at $>$ springeropen.com 